\title{
LOS SEGUROS DE RESPONSABILIDAD CIVIL DE BASE RECLAMACIÓN (CLAIMS MADE) $Y$ LOS DESAFIOS DE SU APLICACIÓN EN EL DERECHO PORTUGUÉS: EL CASO DE LA RESPONSABILIDAD AMBIENTAL
}

\section{THE CLAIMS MADE BASIS \\ CIVIL LIABILITY INSURANCE CONTRACT AND THE CHALLENGES OF ITS APPLICATION IN THE PORTUGUESE LAW: THE ENVIRONMENTAL LIABILITY CASE}

\author{
PATRÍCIA ASSUNÇÃO SOARES* \\ Fecha de recepción: 18 de noviembre 2019 \\ Fecha de aceptación 30 de noviembre 2019 \\ Disponible en línea: 30 de diciembre 2019
}

Para citar este artículo/To cite this article

\begin{abstract}
Assunção Soares, Patrícia, Los seguros de responsabilidad civil de base reclamación (claims made) y los desafíos de su aplicación en el Derecho Portugués: el caso de la responsabilidad ambiental, 51 Rev.Ibero-Latinoam.Seguros, 127-154 (2019). https://doi.org/10.11144/Javeriana.ris51.srcb
\end{abstract}

doi:10.11144/Javeriana.ris51.srcb

\footnotetext{
Abogada del Colegio de Abogados de Portugal; Doctoranda en Derecho Privado en la Facultad de Derecho de la Universidad Nova de Lisboa; Ganadora del Premio Internacional CILA (Comité Ibero-Latinoamericano AIDA) a la Excelencia Académica "Dr. Isaac Halperin" con el trabajo presentado intitulado "Los seguros de responsabilidad civil de base reclamación (claims made) y los desafíos de su aplicación: el caso de la responsabilidad ambiental" que corresponde a la Disertación de Maestría; Máster en Derecho y Gestión por la Facultad de Derecho de la Universidad Nova de Lisboa y NOVA School of Business and Economics; Postgrada en Derecho de Gobierno Corporativo por la Facultad de Derecho de la Universidad de Lisboa (Centro de Investigación de Derecho Privado); Licenciada en Derecho por la Facultad de Derecho por la Universidad de Lisboa. https://orcid.org/0000-0003-0101-3156.
} 


\section{RESUMEN}

El propósito de este estudio es investigar lo contracto del seguro de responsabilidad civil de base reclamación y los desafíos que plantea su aplicación, aplicándolo al instituto de la responsabilidad ambiental, en el sistema legal portugués. La opción por el instituto y la relevancia del su análisis se justifica debido a la naturaleza de los siniestros y daños que poden surgir de la responsabilidad ambiental y por las circunstancias que caracterizan la consecuente reclamación.

En una breve descripción, nuestro estudio se dirige específicamente al análisis del contenido de la cláusula claims made, a la legalidad de su contenido normativo, al impacto de esta cláusula en la determinación del concepto de reclamación, a determinación del momento del ocurrencia da misma y su alcance objetivo y subjetivo, terminando con hipótesis prácticas relativas a la responsabilidad ambiental, que nos permiten probar, en la práctica, las conclusiones extraídas en la presente investigación.

Nuestra análisis en el sistema legal portugués resultó en la conclusiones siguientes: el contenido normativo de la cláusula claims made cuando se aplica a los casos relacionados con responsabilidad ambiental que examinamos, no merece censura legal, que el concepto de siniestro se identifica con la exteriorización de los hechos del asegurado para el asegurador y el conocimiento del asegurador y que la determinación del momento del reclamación coincide con la citación judicial del asegurado en caso del reclamación judicial.

Palabras clave: cobertura, contracto del seguro de responsabilidad civil claims made, daños, delimitación temporal, reclamación, responsabilidad ambiental, siniestro. 


\begin{abstract}
The purpose of this study is to investigate the claims made basis civil liability insurance and the challenges of its practical application in the Portuguese legal system, namely in the context of environmental liability. The choice of this legal regime and the relevance of its analysis is due to the nature of claims and damages that may arise of environmental liability and the circumstances that characterize the consequent claim.

Our study is essentially focused in the the following topics: (i) the content of the claims made clause, (ii) the legality of its normative content, (iii) the impact of this clause in determining the concept of accident, (iv) the determination of the moment of claim and its objective and subjective scope and (v) practical hypotheses which allows us to prove our conclusions.

The main conclusions which were drawn after our investigation are the following: (i) according with Portuguese Law the normative content of the claims made clause should not have any legal censorship when this regime is applied to the environmental liability's cases studied on this paper; (ii) the concept of accident is related with the exteriorization of facts of the insured for the insurer; (iii) the knowledge of the insurer and that the determination of the moment of the claim coincides with the insured's judicial citation in the case of the judicial claim.
\end{abstract}

Keywords: claim, claims made basis civil liability insurance, coverage, damages, environmental liability, losses, temporal delimitation.

\title{
SUMARIO
}

1. LA DELIMITACIÓN TEMPORAL DE LA COBERTURA. 2. LA REALIDAD DEL FACTO DE LA CLÁUSULA CLAIMS MADE. 3. LA COBERTURA RETROACTIVA Y POSTERIOR. 4. LA LEGALIDAD DEL CONTENIDO NORMATIVO. 5. EL IMPACTO DE LA APLICACIÓN EN EL CONCEPTO DE SINIESTRO: EXTERNALIZACIÓN Y CONOCIMIENTO COMO ELEMENTOS IDENTIFICATIVOS. 6. LA DETERMINACIÓN DEL CONCEPTO DE RECLAMACIÓN Y SU ALCANCE. 7. RESPONSABILIDAD AMBIENTAL: HIPÓTESIS PRÁCTICAS. CONCLUSIONES. ANEXOS. 
Todo o conhecimento humano começou com intuiçães, passou daí aos conceitos e terminou com ideias.

EMMANUEL KANT (1724-1804)

\section{LA DELIMITACIÓN TEMPORAL DE LA COBERTURA}

El alcance temporal del contrato se establece en el artículo 37, número 2, letra e) del Régimen Legal del Contrato de Seguro portugués (adelante denominado por RJCS) ${ }^{1}{ }^{2}$ y establece la necesidad de incluir en el contrato el período en que debe ocurrir la reclamación para constituir la obligación del asegurador. A este respecto, la duración del contrato se distingue en el sentido formal, material y técnico ${ }^{3}$. Por duración formal ${ }^{4}$, se entiende el momento de la conclusión del contrato y la vinculación de las partes que dependen del pago de la prima ${ }^{5}{ }^{6}$. La duración material, por su vez, determina el tiempo que el contrato entra en vigencia y el asegurador tiene la obligación de cubrir el riesgo. Por último, la duración técnica está relacionada con el tiempo necesario para tener en cuenta el pago de la prima y la celebración del contrato ${ }^{7}$.

Con efecto, es frecuente que la reclamación pueda ocurrir sin coincidencia entre la duración material y formal del contrato, ocurriendo en la duración material. Destacamos los tres momentos esenciales para la delimitación de la cobertura temporal de lo siniestro $^{8}$ (artículo 139 del RJCS):

\footnotetext{
1 Regime Jurídico do Contrato de Seguro, Decreto-Lei n. ${ }^{\circ} 72 / 2008$, de 16 de abril.

2 A este respecto, el artículo 8 de la Ley del Contrato del Seguro española, Ley 50/1980, de 8 de octubre, tiene la siguiente redacción: "Duración del contrato, con expresión del día y la hora en que comienzan y terminan sus efectos".

3 VEIGA COPO, A. Condiciones en el Contrato de Seguro, Granada, 2008, 2. ${ }^{a}$ edición, p. 142. 4 VEIGA COPO, A. Condiciones en el Contrato de Seguro, Granada, 2008, 2. ${ }^{\text {a }}$ edición, p. 142. 5 Cfr. Artículo 51 número 1 do RJCS, tiene la siguiente redacción: "O prémio é a contrapartida da cobertura acordada e inclui tudo o que seja contratualmente devido pelo tomador do seguro, nomeadamente os custos da cobertura do risco, os custos de aquisição, de gestão e de cobrança e os encargos relacionados com a emissão da apólice."

6 Cfr. Artículo 53 número 1 do RJCS tiene la siguiente redacción: "Salvo convenção em contrário, o prémio inicial, ou a primeira fracção deste, é devido na data da celebração do contrato." 7 VEIGA COPO, A. Condiciones en el Contrato de Seguro, Granada, 2008, 2. a edición, pp. 143 e 348 .

8 Vide, CESERANI, F. "Diritto ed Economia Dell' Assicurazione, Direttori Aurelio D. Candian - Sergio Paci, Vice Direttori Francesco Nanni, 3 - 4, 2007, Giuffrè Editore, p. 799.
} 
a) el hecho donde nace la responsabilidad: el siniestro coincide con el daño cuyo evento surgió durante la vigencia del contrato;

b) la base de ocurrencia de daño (loss occurrence basis): el siniestro coincide con la ocurrencia del daño ${ }^{910}$;

c) la reclamación (claims made) - el siniestro ${ }^{12}{ }^{13}$ coincide con la reclamación ${ }^{14} 1516$.

\title{
2. LA REALIDAD DE FACTO DE LA CLÁUSULA CLAIMS MADE
}

\author{
El artículo 139 número 3 del RJCS establece la validez de la cláusula \\ claims made ${ }^{17}$, asignando la garantía de pago de indemnizaciones
}

9 LACERDA, BRUCK, M. O Seguro dos Administradores no Brasil - O DઐO Insurance Brasileiro, Juruá Editora, p. 222.

10 VISINTINI, G. Trattato della Responsabilità Contrattuale-Applicazioni pratiche e disciplina specifica, Volume Secondo, I Singoli Contrati, CEDAM, 2009, p. 878, o art. 1917. o do Código civil italiano corresponde a lo que comúnmente se llama en la práctica "contrato loss occurrence".

11 Corresponde a la norma general prevista en el artículo 139. ${ }^{\circ}$ do RJCS.

12 REGO, M. L. Colóquio Internacional Governação das Sociedades, Responsabilidade Civil e Protecção dos Administradores - adiantamento dos custos de defesa nos seguros DઐO, 17-05-2018, p. 6, "Nos seguros de responsabilidade civil à base de reclamação, estas integram habitualmente o conceito de sinistro, pelo que não podemos afirmar que ocorreu um sinistro, ou pelo menos que este se completou, antes do surgimento de uma reclamação".

13 Es el llamado problema de long tail risks (siniestros tardíos).

14 Sentencia del Tribunal de la apelación portugues, Tribunal da Relação do Porto, Proceso número 9108/16.0T8PRT-A.P1, 09-11-2017: “(...) a delimitação temporal da garantia do seguro reporta-se não à data da verificação do facto causador do prejuízo, mas antes à data da sua reclamação, diferentemente do que acontece nas apólices de seguro denominadas de ocorrência, que apenas admitem a indemnização quando o facto causador do dano se verifica na vigência do contrato de seguro".

15 RAMOS, Maria Elisabete. O Seguro de Responsabilidade Civil dos Administradores Entre a exposição ao risco e a delimitação da cobertura, Almedina, 2010, p. 457, "na linguagem seguradora IBNR (Incurred But Not Reported), refere-se aos sinistros ocorridos dos quais segurador não teve conhecimento".

16 RAMOS, Maria Elisabete, O Seguro de Responsabilidade Civil dos Administradores Entre a exposição ao risco e a delimitação da cobertura, Almedina, 2010, p. 457, citando María Ángeles Calzada Conde, "La Delimitación..., p. 47, "É para o mercado segurador dar resposta a este tipo de sinistros que surgem as cláusulas claims made. Com esta apólice, o segurador não fica obrigado à prestação no caso de a reclamação não ser formulada durante a vigência da apólice ou dentro do prazo posterior, ainda que o dano de terceiro se tenha produzido".

17 REGO, M. L. Colóquio Internacional Governação das Sociedades, Responsabilidade Civil e Protecção dos Administradores - adiantamento dos custos de defesa nos seguros D\&O, 17/05/2018, p. 3. 
resultantes de daños desconocidos de las partes, ocurridos durante la vigencia del contrato, incluso si la reclamación se presenta al año siguiente $^{18}$. Concluimos que existen dos elementos esenciales de esta cláusula: la intención de indemnización del asegurado y la coincidencia de la reclamación con el siniestro.

Estas cláusulas proporcionan al asegurado la posibilidad de participar el siniestro al asegurador dentro de un período después del cual el asegurador no permanecerá obligado a pagar una indemnización. Así, la cláusula claims made no puede considerarse simplemente como una forma de lo asegurador hacer valer su propia voluntad como un "capricho" o una mera obstinación ${ }^{19}{ }^{20}$.

Consideramos que la ley portuguesa debería prever un concepto de reclamación. La consecuencia práctica es que las partes tienen diferentes interpretaciones sobre el concepto y el momento en que se considera efectuada la reclamación, lo que conduce a un mayor número de litigios. Con efecto, el legislador no debe restringir la libertad contractual de las partes, sino que debe proporcionarles un concepto de dónde partir, dando mayor estabilidad al régimen.

En nuestra opinión, la reclamación es la comunicación realizada por el tomador o asegurado ${ }^{21}$, en la cual el asegurador tiene conocimiento del

18 De conformidad con el artículo 13 del RJCS, solo tiene la calidad de una norma relativamente imperativa, lo que significa que se puede establecer un régimen más favorable para el tomador del seguro, el asegurado o el beneficiario de la provisión del seguro.

19 Lo Tribunal de Milano, en una sentencia da Corte di Cassazione de 18/03/2010, consideró que las clausulas claims made podrían ter dos vertientes: pura, cuando el tema de las reclamaciones por daños recibidos durante el período de la póliza ocurre en relación con los hechos ocurridos en la década anterior; o mixta, que al limitar el efecto retroactivo del hecho relevante limitaría en última instancia el alcance de intervención de la empresa. Este sería el caso si la cláusula que excluye la cobertura se produjo durante dos o tres años (o incluso más) antes de la celebración del contrato, siempre que la rescisión del tercero debe llegar al asegurado durante la vigencia del contrato. . Probablemente en estas hipótesis se determina una limitación de responsabilidad que reduce el plazo.

20 Vide BOGLIONE, A. La Riassicuriazone, pp. 556-560, "o surgimento desta necessidade impositiva surgiu na década de oitenta, no mercado segurador londrino, quando os seguradores começaram a aceitar, precipitadamente, resseguros quanto a riscos de seguradores americanas (...) tornou mais rápida e apelativa a ativação do sistema de claims made, em detrimento do critério da occurence basis".

21 Vide sentencia de la Suprema Corte de Jersey, en caso Zuckerman vs. União Nacional Fire Ins. Co. (1985): “(...) a notificação de reclamação ou ação judicial ocorre quando o segurado tomar conhecimento de qualquer ato ou omissão que possa ser razoavelmente esperado para ser a base de uma ação judicial ou processo coberto por este instrumento. A notificação por escrito 
siniestro, y la fecha de la reclamación se identifica con la fecha en que el asegurador toma conocimiento del siniestro y en el que se considera realizada la reclamación. Consideramos que el criterio para determinar el concepto de reclamación se identifica con el "conocimiento" del siniestro ${ }^{22}$.

Por lo tanto, distinguimos la reclamación en sí de la carga del asegurado para notificar al asegurador sobre las circunstancias que pueden conducir a reclamaciones futuras ${ }^{23}{ }^{24}$. La primera es una activación directa de la cobertura y la segunda consiste en una mayor probabilidad de si verificar la reclamación ${ }^{25}$.

\section{A COBERTURA RETROACTIVA Y POSTERIOR}

Las cláusulas de cobertura retroactiva corresponden a un período acordado por las partes durante el cual se cubren los eventos que causan daños antes de la fecha de vigencia del contrato, si estos hechos dieren lugar a reclamaciones durante el período del contrato ${ }^{26}$.

La ley portuguesa admite el reconocimiento de situaciones en las cuales se encuadra el riesgo putativo ${ }^{27}$, aceptando la validez de la cobertura de un riesgo pasado, reconociendo su protección en el ordenamiento jurídico mediante acuerdo entre las partes.

deve ser realizada em nome do segurado ao segurador assim que possível, juntamente com as informações mais completas possível".

22 CANNON, M. Mc GURK, B. Professional Indemnity Insurance, Second Edition, Oxford University Press, no entendimiento de American Insurance Co vs Fairchild Industries Inc., p. 24, La definición de la cláusula claims made adoptadas por las aseguradoras de los Estados Unidos consiste en informar al asegurado o a un tercero al asegurador por los daños que entran dentro de los riesgos cubiertos por la póliza.

23 Cfr. American Continental Ins. Co. vs. Steen, Supreme Court of Washington, En Banc., 14/05/2004, en el caso de las pólizas de base reclamación, si el asegurador no recibe ninguna reclamación dentro del período de la póliza, el asegurador no se considera responsable.

24 CANNON, M. Mc GURK, B. Professional Indemnity Insurance, Second Edition, Oxford University, pp. 21 e ss, considera que la obligación de notificar está vinculada a la consecuencia de adoptar una poliza claims made.

25 Es una declaración de agravamiento del riesgo.

26 Para este propósito, es esencial que el evento dañoso sea desconocido de las partes en el momento de la conclusión del contrato (artículo 44, número 2 del RJCS).

27 Sentencia del Tribunal de la apelación portugués, Tribunal da Relação de Lisboa, 26/05/1987, Proceso número 0005804. 


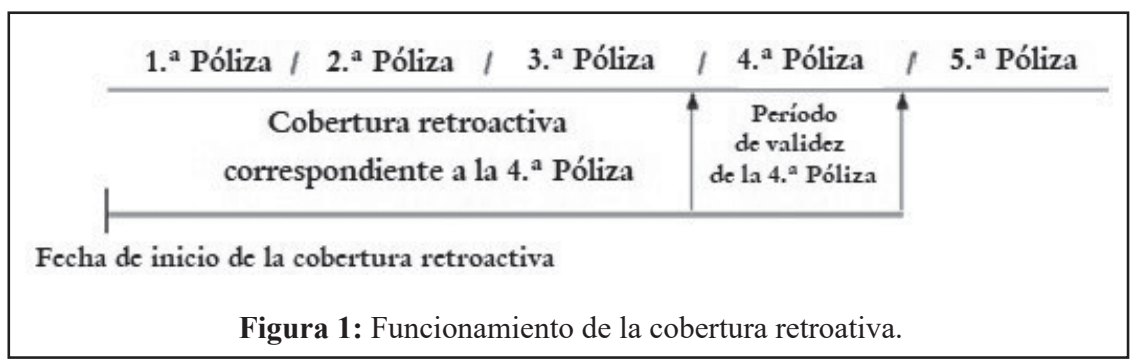

Fuente: Adaptado ${ }^{28}$.

Las cláusulas de cobertura posteriores se refieren a las reclamaciones ocurridas después de vencido el contrato ${ }^{29}$, por hechos ocurridos durante el período del mismo, pero que se materializan en el año siguiente ${ }^{30}$ 31. La aseguradora extiende la cobertura evitando uno vacío entre el final del contrato anterior y el inicio de uno nuevo ${ }^{32}$. Dado que estas cláusulas corresponden a un acuerdo entre las partes que estipula la extensión de la cobertura, caducan al final dese período y por eso se les aplica el régimen de caducidad ${ }^{33}$.

28 Adaptado de MENDES, C., ZETTEL, C., COSTA, M. "Revista Brasileira de Risco e Seguro - As apólices à base de reclamação no Brasil”, p. 155.

29 Con respecto al vencimiento del contrato, también surge la pregunta de si, en los casos en que el tomador del seguro no paga la prima, ¿ las reclamaciones realizadas durante el plazo de la cobertura posterior deben considerarse cubiertas? La cobertura de riesgo depende del pago previo de la prima, de conformidad con el artículo 59 del RJCS. Si no se ha pagado la prima, el asegurado no tiene derecho a la cobertura contratada. Dado que el pago de la prima es una condición formal del contrato, si el asegurado no ha pagado la prima, el asegurador no está obligado a cubrir un reclamo durante la cobertura posterior. Tenga en cuenta que, según los términos del artículo 433 del Código Civil, la resolución se equipara, en cuanto a sus efectos, a la nulidad o nulidad del negocio legal. Además, de conformidad con el Artículo 61 número 1 del RJCS, la falta de pago de la prima inicial o la primera cuota en la fecha de vencimiento terminará automáticamente el contrato desde la fecha de su conclusión.

30 https://www.fortunylegal.com/blog/las-clausulas-claim-made-en-el-seguro-deresponsabilidad-civil.html.

31 HERMIDA, A. Las Cláusulas "claims made" en los seguros de responsabilidade civil patronal: jurisprudência reciente de la Sala de lo Social del Tribunal Supremo, in http://ajatapia. com/2016/05/las-clausulas-claims-mae-en-los-seguros-de-responsabilidad-civil-patronaljurisprudencia-reciente-de-la-sala-de-lo-social-del-tribunal-supremo/.

32 PÉREZ, C. R. Contrato de Seguro - Introducción a la Ley 50/1980, Lisboa, Editora Juruá, 2017, p. 58, “a cláusula de cobertura posterior exige duas condições para que o segurador seja obrigado ao pagamento de indemnização: a ocorrência do dano durante a cobertura do contrato e a reclamação do terceiro se verifique no prazo do contrato ou, no máximo, durante o ano imediatamente seguinte. A necessidade de concordância destes dois requisitos constitui uma verdadeira alteração contratual do curso normal do contrato uma vez que se não fosse pela aplicação dessa cláusula o segurador responderia se se verificasse a primeira condição".

33 Sentencia del Tribunal de la apelación portugues, Tribunal da Relação de Guimarães, 15-02-2006, Proceso número 2480/05-2. 


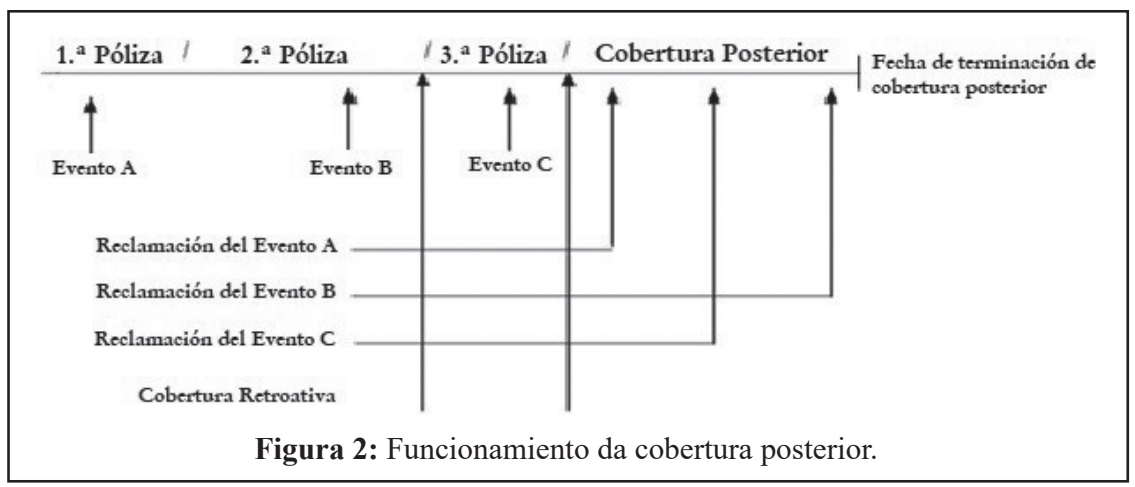

Fuente: Adaptado $^{34}$.

\section{A LEGALIDAD DEL CONTENIDO NORMATIVO}

La ley portuguesa prevé la posibilidad del tercero perjudicado puede reclamar directamente al asegurador (artículo 140, números 2 y 3 y artículo 146, número 1 del RJCS) ${ }^{35}$, aplicando los plazos de prescripción de lo Código Civil portugués (adelante CC) ) $^{36}$ de tres años ${ }^{38}{ }^{39}$. Este es

$34 \quad$ Adaptado de MENDES, C., ZETTEL, C., COSTA, M. Revista Brasileira de Risco e Seguro - As apólices à base de reclamação no Brasil, (...), pp. 155 e ss.

35 REGO, M., L. Contrato de Seguro e Terceiros - Estudo de Direito Civil, maio de 2010, Coimbra Editora, pp. 670 e ss, "não existe uma previsão pela qual se possa concluir pela ação direta generalizada em todos os seguros de responsabilidade civil. (...) Considera que, reconhecer um direito de ação direta ao terceiro lesado contra o segurador, permite-se que, por exemplo no caso do seguro de responsabilidade civil por acidentes de viação, a questão da existência ou não de responsabilidade do segurado se discuta e se decida unicamente entre o segurador e o terceiro lesado, ou entre estes perante o tribunal. Tal pode configurar uma sujeição para o segurado, já que vê o seu poder de intervenção fortemente limitado e por outro lado, uma imunidade perante o terceiro lesado porque lhe é "retirado" o poder de exigir a indemnização a este (que era o próprio responsável).”

36 Por remisión de artículo 145 del RJCS.

37 Cfr., RODRIGUES, Tânia Matos. A Prescrição nos seguros de responsabilidade civil de base reclamação, 2018, p. 61: “(...) é permitido ao lesado exercer o seu direito de ação face ao segurador, ficando sujeito ao regime prescricional previsto nos art. $306 .^{\circ}$ e ss e $498 .^{\circ}$ do CC".

38 El Código Civil español establece la coincidencia del período máximo de reclamo con el período de limitación para la acción de responsabilidad civil, en el art. 1968, párrafo 2 y art. 73 de la Ley española de contratos de seguro (Ley 50/1980, de 8 de octubre).

39 Por la conjugación de los artículos 4 y 11 del RJCS, el contrato de seguro se limita a los requisitos de la ley general y, teniendo en cuenta estas disposiciones, la ley civil se aplica como alternativa. 
un período no prorrogable (de acordó con el artículo 300 del CC) $)^{40} 41$ y su incumplimiento conduce a la nulidad del contrato.

El plazo de un año a que se refiere el artículo 139 número 3 del RJCS es un plazo de delimitación temporal de la responsabilidad del asegurador, pero no es un plazo de prescripción, tiendo en cuenta que no excluye las disposiciones de la prescripción ${ }^{42}$, antes se extingue con el transcurso del tiempo, siendo un plazo de caducidad, de conformidad con el artículo 298 del $\mathrm{CC}^{43}$.

La extensión temporal de la cobertura es un derecho que está totalmente disponible para las partes ${ }^{44}{ }^{45}$ y que pueden ser regulados por ellas con respecto al curso del tiempo ${ }^{46}$.

Si el contrato de seguro estipula la posibilidad de una acción directa, lo tercero perjudicado tiene el plazo previsto en las reglas de prescripción ${ }^{47}$

40 LIMA, Pires de, VARELA, Antunes. Código Civil Anotado, Vol. I, 4. ${ }^{a}$ Edição revista e atualizada, Reimpressão, Coimbra Editora, 2011, p. 274, Ponto 2 da Anotação: "A proibição estabelecida na lei e a solução prescrita para a sua violação (nulidade do negócio) explicamse pelas razões de interesse e ordem pública (...) destinado a tutelar a certeza do direito e a segurança do comércio jurídico".

41 PRATA, Ana. Código Civil Anotado, Almedina, 2017, Vol. I, p. 375, Comentário de Rita Canas da Silva ao art. 300. ${ }^{\circ}$, “(...) A natureza imperativa ou inderrogável é decorrência da difícil tarefa, tentada pelo regime legal, de harmonização dos valores conflituantes".

42 MORGADO, J., P., RIBEIRO, M., E., TORRES, L., C., OLIVEIRA, A., C., MARTINEZ, P. , R., VASQUES, J. Lei do Contrato de Seguro Anotada, Almedina, 2016, Comentário de José Vasques, p. 449.

43 Las partes solo acuerdan el plazo dentro del cual existirá la cobertura del asegurador y lo obligarán al pago de una compensación, estipulando períodos dentro del alcance de sus derechos disponibles, después de los cuales expira el derecho a la cobertura.

44 Vide, PRATA, Ana, (Coord.), Código Civil Anotado, Almedina, 2017, Comentário de Ricardo Bernardes, p. 400 e ss.

45 PROENÇA, José Brandão, FERNANDES, Luís Carvalho (Coord.). Comentário ao Código Civil, Parte Geral, Universidade Católica Editora, Anotação ao Ac. STJ de 26/06/1970, RLJ n. ${ }^{\circ}$ 3453, pp. 770 e ss. "parece que as caducidades convencionais não devem considerarse válidas quando se tratar de matéria subtraída à disponibilidade das partes, quando houver fraude à lei sobre prescrição ou quando elas tornares excessivamente difícil o exercício do direito, embora seja sempre necessário verificar se no caso concreto a estipulação da cláusula constitui uma fraude à lei".

46 Cfr. LEITÃO, Luís Menezes. Direito das Obrigações, Vol. II, 2. ${ }^{a}$ Edição, 2003, p. 102, La caducidad, establece que "(...) consiste na extinção em virtude da ocorrência de um facto jurídico stricto sensu (...) sendo o exemplo mais comum o decurso do tempo".

47 En conformidade com la sentencia de lo Supremo Tribunal de Justicia portugues, 22-10-2015, Proceso número 273/13.9YHLLSB.L1.S1, "A caducidade não tem por fundamento primeiro a proteção do sujeito passivo mas sim o valor da certeza e segurança dos direitos. (...) O estabelecimento legal de um prazo de caducidade para o exercício de um direito não afasta a 
de tres años ${ }^{48}$ y su derecho permanecerá una vez transcurrido el plazo previsto en el artículo 139 número 3 del RJCS ${ }^{49} 50$.

Por lo tanto, tenemos el plan de la prescripción y el plan de la caducidad, que es un período estipulado contractualmente dentro del cual el asegurador está obligado a cumplir con la obligación del asegurado. Consideramos que estos dos planes son compatibles porque dicen respecto a plazos diferentes, que regulan derechos diferentes ${ }^{51}$.

En cuanto a la asimetría prestacional, la naturaleza del contrato de seguro justifica la naturaleza legítima de las cláusulas claims made, convirtiéndolo en una verdadera imposición. Del lado del asegurador está la obligación de indemnizar y del lado del asegurado está el derecho a indemnización. Así, existe una necesidad práctica imperiosa de, en primero lugar, no eternizar la vinculación del asegurador y, en segundo lugar, evitar que el asegurador excluya la cobertura de siniestros, lo que resulta en una reciprocidad y compensación mutua entre las partes, lo cual es un sinalagma jurídico ${ }^{52}$. Concluimos que las cláusulas claims made no

aplicabilidade do prazo geral da prescrição "por não exercício (...)“o estabelecimento legal de um prazo de caducidade para o exercício de um direito não afasta a aplicabilidade do prazo geral da prescrição", disponível in http://dgsi.pt/jstj.nsf/ /32F441C49A047C8A80257EE6005813FF.

48 REGO, M. L. Contrato de Seguro e Terceiros-Estudo de Direito Civil, 2010, Coimbra Editora, pp. 680 e seguientes.

49 Sentencia del Tribunal de la apelación portugues, Tribunal da Relação do Porto, Proceso número 721/08.0TVPRT-A.P1, 06-07-2009 y sentencias del Tribunal de la apelación, Tribunal da Relação de Lisboa, Proceso número 1612/11.2TVLSB-A.L1-2, 29-09-2016 e Proceso número 418/13.9TVLSB, 11-01-2018.

50 El número 3 del artículo 139 del RJCS, al proporcionar "en el año siguiente a la expiración del término" se refiere a un término que se aplica al asegurado o también a las "partes" del tercero perjudicado sin especificar a qué partes se refiere. Entendemos que esta disposición está dirigida y regula el período de reclamación del asegurado que tiene un año para presentar su reclamación, que delimita el período de tiempo en que el asegurador asume la responsabilidad del pago de la compensación. El propio RJCS regula en otras disposiciones específicas el ejercicio del derecho de la parte perjudicada. Por lo tanto, se entiende que la sistematización de la ley está diseñada para regular dos posiciones jurídicas diferentes y con sus propias especificidades y regímenes.

51 MELLO, Sérgio Barroso. Validade da cláusula claims made perante o Direito Brasileiro, Set. 2005, "Para evitar a abusividade, as cláusulas devem ser redigidas de forma clara e compreensível, onde o segurado decide aquela cobertura contratual que lhe convier mais justa e econômica, e onde se verifica que a cláusula "claims made" é nuclear ao contrato de seguro e à cobertura pretendida.", disponible in http://pellon-associado.hospedagemsites.ws/portal/ filesPublicacoes/Artigos/smello_12.pdf.

52 Sobre la validez da claims made, vea los siguientes ejemplos de decisiones de los Estados Unidos que han confirmado la validez inequívoca de esta cláusula: Scarborough vs. Travelers Ins. Co.; James \& Hackworth vs. Continental Cas. Co., (1980); Brander vs. Nabors; Gulf Ins. Co. 
interfieren con el período de prescripción de lo tercero perjudicado ni causan un desequilibrio contractual en el sistema legal portugués.

\section{EL IMPACTO EN EL CONCEPTO DE SINIESTRO: EX- TERNALIZACIÓN Y CONOCIMIENTO COMO ELEMENTOS IDENTIFICADORES}

El siniestro es el evento que determina el surgimiento de la responsabilidad del asegurador cuando el tercero ha expresado su intención de ser indemnizado ${ }^{53}$.

Entiende Fernando SANCHÉZ CALERO ${ }^{54}$ que dos teorías se oponen: la que identifica el siniestro con el hecho dañoso y la que establece que lo siniestro solo se materializa con la presentación de la reclamación ${ }^{55} 56$.

vs. Dolan, Fertig e Curtis, (1983); Livingston Parish School Bd. vs. Fireman's Fund Am. Ins. Co., (1973); Stine vs. Continental Cas. Co., Lehr vs. Professional Underwriters, (1941); Gereboff vs. Home Indem. Co., (1978); Las decisiones que afirmaban la validez de las reclamaciones constituían políticas simplemente al aplicarlas: Hunter vs. Office of Health Servs. Qualidade Ambiental, (1980); Samuel N. Zarpas, Inc. vs. Morrow; VTN Consol., Inc. vs. Northbrook Ins. Co., Inc (1979); San Pedro Properties, Inc. vs. Sayre \& Toso, Inc., (1962); Graman vs. Continental Cas. Co., (1980); Breaux vs. St. Paul Fire \& Marine Ins. Co., (1976); JM Brown Constr. Co. vs. D \& M Mechanical Contractors, Inc., (1969) y otros tribunales que no cuestionaron la validez general de las reclamaciones hicieron pólizas pero interpretaron los términos y condiciones de la póliza para determinar la legalidad de la cobertura del contrato de seguro: Hoyt vs. São Paulo Fire \& Marine Ins. Co., (1979); Troy \& Stalder Co. vs. Continental Cas. Co., (1980); Manacre Corp. vs. First State Ins. Co., (1979); Cas Continental. Co. vs. Enco Assocs., Inc., Mich. (1975), todos los casos citados en la decisão do caso Zuckerman vs. National Union Fire Ins. Co. (1985).

53 GARRIGUES, J. Contrato de Seguro Terrestre, Segunda Edición, Madrid MCMLXXXIII citando ANGELONI na p. 363), p. 370.

54 CALERO, F. S. Ley de Contrato de Seguro-Comentarios a la Ley 50/1980, de 8 de octubre, y a sus modificaciones, Thomson Arazandi, 3. ${ }^{a}$ Edición, p. 1318 e siguientes, la primera tesis fue apoyada por la doctrina alemana e italiana y la segunda por la doctrina francesa (presente hoy en el actual artículo 124.1 de la Ley francesa de contrato de seguro).

55 GARRIGUES, J. Contrato de Seguro Terrestre, 1982, cita, en defensa de esta concepción, el autor GIERKE (p. 299) para quien "o seguro de responsabilidade civil concede proteção contra as consequências da reclamação de um terceiro. De acordo com a lei alemã, o sinistro coincide com a reclamação do terceiro. (...) A peculiaridade do seguro de responsabilidade civil consiste no facto de não ser necessário que o sinistro ocorra no tempo do seguro, porque a reclamação do terceiro pode fazer-se depois".

56 Otra es la comprensión de la jurisprudencia italiana que considera que, para los efectos de la responsabilidad civil, la reclamación es el hecho ilícito que da lugar a la reclamación y no la reclamación en sí misma y afirma que las cláusulas de reclamaciones derogan el artículo 1917 del CC y limita la responsabilidad del asegurador agravando la posición del asegurado. (...) El contrato del seguro de responsabilidad civil que incorpora la cláusula claims made no se encuadra a la disposición del artículo 1917 del CC italiano, pero constituye un contrato atípico 
Por lo tanto, la pregunta es: ¿cuál es el concepto de siniestro en el caso de daños diferidos?

La ley portuguesa ${ }^{57}$ no responde a esta cuestión proporcionando una noción general de siniestro sin especificarla de acuerdo con el tipo de daño ${ }^{58} 59$.

Entendemos que el concepto de siniestro depende del tipo de daño y el momento en que ocurre. Así, en los siniestros tardíos, la ocurrencia de daños diferidos no coincide entre la duración material y la duración formal del contrato, lo que significa que el siniestro, muchas veces, ocurre fuera del plazo de vigencia del contrato.

La cuestión es reconocer en una secuencia de hechos, aquel en el que se considera producido lo siniestro y cómo podemos identificarlo con la reclamación.

Consideramos que el siniestro se materializa por una pluralidad de hechos que se prolongan temporalmente de forma parcelar y que lo que desencadena la reclamación es el último hecho que perjudica el bien digno de cobertura. Este es el momento en que el daño se materializa y se unifica.

Por lo tanto, el asegurador solo sabe que el siniestro ocurrió porque le fue transmitido, por eso se considera que es la externalización de los hechos del asegurado al asegurador, y su conocimiento de ellos, que constituyen los criterios de definición y concretización del siniestro, que coincide con la reclamación. La prueba de que se mencionó es que, si no hay reclamación y el asegurado no informa el siniestro a lo asegurador, no hay cobertura porque el asegurador no ten conocimiento de los daños y todo sucede como si no existiese un siniestro ${ }^{60}$.

(considerado válido de conformidad con el artículo 1322 del CC italiano); Sentencia de la Corte Suprema di Cass., 15/03/2005, pp. 15 e 19.

$57 \quad$ Artículo 99 del RJCS.

58 MORGADO, J., P., RIBEIRO, M., E., TORRES, L., C., OLIVEIRA, A., C., MARTINEZ, P. , R., VASQUES, J. Lei do Contrato de Seguro Anotada, Almedina, 2016, Comentário de Arnaldo Costa Oliveira, p. 355, nos enfrentamos a una noción "minimalista, neutra e aberta", es necesario determinar un criterio que nos permita establecer una definición para el concepto de siniestro que produce daños diferidos.

59 Cfr. COPO, VEIGA, A. Tratado del Contrato de Seguro, Civitas, Thomsom Reuters, p. 182, "Não é fácil nem definitivo, balizar os contornos reais da noção de sinistro. O silêncio do Legislador dificulta o caminho. A teoria é díspar como também o são os entendimentos jurisprudenciais."

60 El criterio de conocimiento presentado aquí coincide con el criterio de "conocimiento" al que se refiere el RJCS en varias normas, y se puede concluir que este es un elemento ampliamente utilizado por el propio sistema de la ley. $25,26,30,44,55,83$, etc. 


\section{LA DETERMINACIÓN DEL MOMENTO DE LA RECLA- MACIÓN Y SU ALCANCE}

La cuestión es saber cuál es el momento relevante ${ }^{61}$ donde el reclamación se considera efectuada en el dominio judicial (cuando hay una demanda) y extrajudicial (notificación por escrito).

En el plano judicial, si el asegurador tiene conocimiento de la existencia de la reclamación solo en la fecha de la citación en la que se le llama a intervenir, no puede conocer la fecha en que se presentó la reclamación, si solo conocía la reclamación cuando fue convocado judicialmente como interviniente. Por lo tanto, el argumento de la no cobertura es válido, porque cuando lo asegurador se dio cuenta de la reclamación, la póliza ya no estaba en vigor.

Consideramos que la fecha relevante en el dominio judicial portugués, a los efectos de considerar la reclamación presentada, es la fecha de citación judicial del asegurado, suponiendo que el asegurador no haya recibido previamente ninguna notificación extrajudicial. Es el asegurado, quien es responsable de transmitir al asegurador que hay una demanda en curso por la cual ha sido citado y las circunstancias del caso, y en esta fecha el asegurador debe considerar que la reclamación ha ocurrido ${ }^{62}$.

En cuanto al contenido de la reclamación, es a través de su análisis que la aseguradora evalúa las circunstancias de la reclamación y determina su responsabilidad. Cuanto mayor sea el grado de especificación del contenido objetivo de la reclamación, más fácil será para el asegurador activar la cobertura contratada. Por lo tanto, cuanto más calificado sea el conocimiento de la aseguradora sobre el siniestro, mejor será el desempeño contractual de la cobertura.

Subjetivamente, la cuestión es saber cuál es la relevancia cuando se hace la reclamación del asegurado para lo tomador del seguro o del

61 VASQUES, J. Contrato de Seguro - Notas para uma teoria geral, 1999, p. 300, considera que "o momento em que se dá por realizada a reclamação corresponde ao momento em que o segurador tem conhecimento do sinistro. Analogamente, está a par com a participação do sinistro. Considera-se por isso um ónus jurídico no sentido em que da reclamação (momento em que o segurador tem conhecimento de que ocorreu um sinistro) depende a obtenção da prestação indemnizatória do segurador, sendo que a sua inobservância a poderá excluir".

62 Este argumento tiene soporto legal en la aplicación del principio de estabilidad de la instancia incorporada en el artículo 260 del Código de Processo Civil, ya que es en esta etapa del procedimiento judicial que el tribunal establece las personas, el pedido y su causa. 
tercero perjudicado para el asegurado ${ }^{63}$. Ambas las comunicaciones corresponden a una primera etapa de conocimiento, pero en ninguno de los casos se considera concretizada la reclamación. Consideramos que, solo el conocimiento del asegurador es relevante para los efectos de considerar la reclamación ya que el asegurador es la parte contractual que asume la obligación de indemnizar. En consecuencia, la existencia de una reclamación en ambos los casos dependen de si las partes han informado al asegurador y solo entonces se considera que la reclamación ocurrió. Así, todas las comunicaciones que no incluyen al asegurador constituyen una primera etapa de conocimiento, pero que la reclamación solo se materializa con el conocimiento del asegurador.

\section{LA RESPONSABILIDAD AMBIENTAL: HIPÓTESIS PRÁCTICAS}

\section{- Hipótesis 1:}

La empresa A practica descargas en un río para la gestión de sus residuos $^{64}$. En 2018, la empresa B presentó una demanda contra la empresa A por daños derivados de su actividad. Los últimos dos contratos de seguro celebrados por la empresa A fueron los siguientes: con la Aseguradora 1, en febrero de 2016, durante un año, cubriendo las reclamaciones presentadas hasta un año después (febrero de 2018); y con la Aseguradora 2, en abril de 2017, durante dos años, cubriendo las reclamaciones presentadas hasta un año después (abril de 2020) ${ }^{65}$.

La pregunta es la siguiente: ¿cuándo se considera efectuadala reclamación?

En este caso, no hay coincidencia entre la duración formal y material del contrato y, por lo tanto, al referirnos al contrato vigente en la presentación de la reclamación, nos referimos a la dimensión material que lo determina el tiempo que el contrato produce efectos y en que la aseguradora está vinculada a su obligación de indemnizar.

63 En ambos casos hay una externalización de los hechos que conforman el contenido objetivo de la queja.

64 En vista de la obligación prevista en el Artículo 22, número 2 y el Anexo III del DL 147/2008, de 29 de julio, el Régimen Legal de Responsabilidad por Daños Ambientales, ha celebrado varios contratos de seguro.

65 El momento del reclamo coincide con un elemento de externalización y conocimiento por parte de la parte asegurada / lesionada al asegurador, en caso de reclamo si es judicial o extrajudicial. El daño presentado surge de un reclamo tardío y, por lo tanto, el reclamo se identifica con la fecha de conocimiento por parte de la aseguradora. 
Entendemos que el momento de la proposición de la acción y la citación deben articularse para determinar el momento de la reclamación ${ }^{66}{ }^{67}$. Por lo tanto, la cobertura posterior del contrato con la Aseguradora 1 cesó en el momento en que comenzó la cobertura de la Aseguradora 2 y, por esa razón, es el contrato con la aseguradora 2 el que cubre el siniestro.

\section{- Hipótesis 2:}

El asegurado, na conclusión del contrato con la Aseguradora 2, conocía que tiña ocurrido un hecho dañoso y no lo transmitió a la Aseguradora.

La garantía de cobertura de la reclamación presupone que los eventos nocivos son desconocidos para las partes (Artículo 139, número 3 del RJCS). Así, entendemos que el contrato no tiene efecto, esencialmente, por dos razones: la primera es la nulidad, según el artículo 44 números 1 y 2 del RJCS, que establece que el contrato sufre de nulidad si el asegurado conocer os hechos que causan la cesación del riesgo, lo que revela su mala fe. ${ }^{68}$; y la segunda es la anulabilidad, a través del envío al asegurador por lo asegurado de una declaración, en conformidad con los artículos 24 y 25 del RJCS teniendo en cuenta que en la declaración inicial de riesgo, el asegurado no declaró todas las circunstancias que conocía e que sabía que eran necesarias.

\section{CONCLUSIONES}

1. La cláusula claims made circunscribe la obligación del asegurador, a presentación de la reclamación por lo asegurado durante la vigencia del contrato, independientemente del hecho que ocasione el daño.

66 De conformidade con artículo 259, número 2 do Código de Processo Civil “( ...) o ato da propositura não produz efeitos em relação ao réu senão a partir do momento da citação".

67 FREITAS, José Lebre de; ALEXANDRE, Isabel. Código de Processo Civil Anotado, Vol. 1. ${ }^{\circ}$, Coimbra Editora, 3. ${ }^{a}$ Edição, "Com a citação, o ato de propositura da ação ganha eficácia em face do réu, que com ela fica constituído como parte, e a instância estabiliza nos seus elementos subjetivos e objetivos."

68 REGO, M. L. Temas de direito dos seguros, O risco e as suas vicissitudes, p. 3, "A má fé a que a lei faz referência é a má fé subjetiva. (...) o caso de inexistência do risco prende-se, com uma insuficiente tomada de consciência de que, desconhecendo o segurador os elementos que lhe permitem concluir pela inexistência do risco, este foi por ele suportado, (...) nos mesmos termos em que o segurador suportou todos os outros riscos. Em caso de dolo do tomador do seguro ou do segurado com o propósito de obter uma vantagem, aplicar-se-á ainda o regime da fraude", in https://www.fd.unl.pt/docentes_docs/ma/MLR_MA_13700.pdf. 
2. La reclamación es la comunicación realizada por el asegurado o asegurado, en la cual el asegurador es informado del siniestro.

3. El criterio para determinar el concepto de la reclamación se identifica con lo "conocimiento".

4. El plazo previsto en el artículo 139, número 3 del RJCS es un período de delimitación de la responsabilidad, cuya naturaleza es la caducidad y es compatible con la existencia del plazo de prescripción, que corresponde a lo tercero perjudicado.

5. Existe reciprocidad y compensación mutua entre las partes en el contrato, lo cual constituí un sinalagma jurídico.

6. La externalización de los hechos y el conocimiento son los criterios definidores de los siniestros.

7. El asegurado tiene la carga de informar al asegurador que hay una demanda en curso por la cual ha sido citado judicialmente y es en esta fecha que ocurre la reclamación.

8. Las comunicaciones entre las partes que no incluyen al asegurador constituyen una primera etapa de conocimiento, pero la reclamación solo se concretiza con o conocimiento de lo asegurador de la misma. 
ANEXOS $^{69}$

Póliza "Commercial General Liability" (CGL) efectuada por Insurance Service Office Inc. (ISO), en 1986.

\section{COMMERCIAL GENERAL LIABILITY COVERAGE FORM COVERAGE A. PROVIDES CLAIMS MADE COVERAGE. PLEASE READ THE}

Various provisions in this policy restrict coverage. Read the entire policy carefully to determine rights. duties and what is and is not covered.

Throughout this policy the words "you" and "your" refer to the Named insured shown in the Declarations. The words "we." "us" and "our" refer to the Company providing this insurance.

The word "insured" means any person or organiza. tion qualifying as such under SECTION II - WHO IS AN INSURED.

Other words and phrases that appear in quotation marks have special meaning. Refer to SECTION VI DEFINITIONS.

" SECTIONI - COVERAGES

COVERAGE A. BODILY INJURY AND PROP. ERTY DAMAGE LIABILITY

1. Insuring Agreement.

a. We will pay those sums that the insured becomes legally obligated to pay as damages because of "bodily injury" or "property dam. age" to which this insurance applies. No other obligation or liability to pay sums or per. form acts or services is coverms or per. explicitly provided for under SUPPLEMEN. TARY PAYMENTS - COVERAGES A AND B. This insurance does not apply to "bodily injury" or "property damage" which occurred before the Retroactive Date if any occur. in the Declarations or which occurs after the policy period. The "bodily injury" or "prop. erty damage" must be caused by an "occur. rence " The occurrence" must take place to the cover.sge territory "we will have the right ind duty to detend any "suit" seekin: those damages. 6 ut

(1) The amount we will pay for damages :s Iumited as described in SECTION III.. LIMITS OF INSURANCE:

(2) We may, at our discretion, investigate any occurrence " and settle any claim or "suit" that may result: and

(3) Our right and duty to defend end when we have used up the applicable limit of insur. ance in the payment of judgments or set thements under Coverages A or B or mert Whilexpenses under Coverage $C$

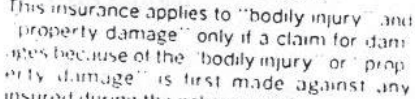
insurist during the policy period
(1) A claim by a person or organization seek. ing damages will be deemed to have been. made when notice of such claim is received and recorded by any insured is received and recorded by any insured or
by us, whichever comes first.

(2) All claims for damages because of "bodily injury" to the same person, including damages claimed by any person or orga nization for care loss of services. death resulting at any time from "bodily injury" will be time from the bodily injury." will be deemed to have been made at the time the first of those claims is made against any insured

(3) All claims for damages because of "prop erty damage" causing loss to the same person or organization as a result of an "occurrence" will be ds a result of an been made at the time the first of those 2. Exclusions. claims is made against any insured.

This insurance does not apply to:

a. "Bodily injury" or "property damage" ex. pected or intended from the standpoint of the insured. This exclusion does not apply "bodily injury" resulting from the use of "bodily injury" resulting from the use of reasonable force to protect persons or prop-
erty.

"Bodily injury" or "property damage" for which the insured is obligated to pay dam. ages by reason of the assumption of liabilit. a contract or agreement. This exclusion not spoly to lrability for damoges

(1) Assumed in a contract or apreem...... is an "insured contract." or

(2) Inat the ansured would have an tro mence ot the contract or agreamer.

c. "Bodily infury" or "property damage ":. which any insured may be held irable $s$;
reason of

(1) Causing or contributing to the intoxic? tion of any person:

(2) The furnishing of alcoholic beverages to.: person under the legal drinking age :under the influence of alcohol: or

(3) Any statute. ordinance or regulatio reiating to the sale. gift. distribution use of alcoholic beverages

liws axitusion applies only if you are :: * . isusintess ot manutacturine distributine se the: siveviny: or furnishung alcoholit bever,at: 
(5) That particular part of real property on which you or any contractors or subcon. tractors working directly or indirectly on your behalf are performing operations, if the "property damage" arises out of those operations: or

(6) That particular part of any property tha must be restored, repaired or replaced because "your work" was incorrectly performed on it.

Paragraph (2) of this exclusion does not apply if the premises are "your work" and were never occupied, rented or held for rental by you.

Paragraphs (3). (4). (5) and (6) of this exclusion do not apply to liability assumed under a sidetrack agreement.

Paragraph (6) of this exclusion does not apply to "property damage" included in the "pro. ducts-completed operations hazard."

k. "Property damage" to "your product" arising out of it or any part of it.

I. "Property damage" to "your work" arising out of it or any part of it and included in the "products-completed operations hazard." This exclusion does not apply if the damaged work or the work out of which the damage arises was performed on your behalf by a subcontractor.

m. "Property damage" to "impaired property" or property that has not been physically injured, arising out of:

(1) A defect, deficiency, inadequacy or dan gerous condition in "your product " or your work: " or

(2) A delay or failure by you or anyone actin on your behalf to perforni a contract o: agreement in accordance with its terms

This exclusion does not apply to the loss of use of other property arising out of sudden and accidental physical injury to "your product" or "you work" after it has been put to its intended use

n. Damages claimed for any loss. cost or ex. pense incurred by you or others for the loss of use, withdrawal, recall, inspection, repair. replacement, adjustment, removal or dis. posal of:

(1) "Your product:"

(2) "Your work:" or

(3) "Impaired property:"

if such product. work. or property is with drawn or recalled from the markel or from use by any person or organization because ot a known or suspected defect. deficiency inadequacy or dangerous ciondition in it
COMMERCIAL GENERAL LIABUTYY COVERAGE FORM

Exclusions c. through n. do not apply to damage by fire to premises rented to you. A separate limit of insurance applies to this coverage as described in SECTION III - LIMITS OF INSURANCE.

COVERAGE B. PERSONAL AND ADVERTIS. ING INJURY LIABILITY

1. Insuring Agreement.

a. We will pay those sums that the insured becomes legally obligated to pay as damages because of "personal injury" or "advertising injury" to which this insurance applies. other obligation or liability to pay sums or perform acts or services is covered unless explicitly provided for under SUPPLEMEN. TARY PAYMENTS - COVERAGES A AND 8 . We will have the right and duty to defend any "suit" seeking those damages. But:

(1) The amount we will pay for damages is limited as described in SECTION III LIMITS OF INSURANCE:

(2) We may investigate and settle any claim or "suit" at our discretion; and

(3) Our right and duty to defend end when we have used up the applicable limit of insur ance in the payment of judgments or settlements under Coverages A or B or mediCal expenses under Coverage $C$

b. This insurance applies to "personal injury" only if caused by an offense:

(1) Committed in the "coverage territory" during the policy period: and

(2) Arising out of the conduct of your business. excluding advertising. publishing. broadcasting or teiecasting done by or for you

c. This insurance applies : 2 adverticing infury only if cuused by an affense committed

(1) In the coverage territory" during the policy period: and

(2) in the course of advertising your goods. products or services.

2. Exclusions.

This insurance does not apply to:

a. "Personal injury" or "advertising injury:"

(1) Arising out of oral or written publication of material. if done by or at the direction of the insured with knowledge of its faisity.

(2) Arising out of oral or written publication ct material whose first publication toor place before the beginning of the policy period.

(3) Arising out of the willful violation of is penal statute or ordinance committed by or with the consent of the insured: or 
COMMERCIAL GENERAL LIABILITY COVERAGE FORM

(4) For which the insured has assumed liability in a contract or agreement. This exclusion does not apply to liability for dam. ages that the insured would have in the absence of the contract or agreement.

b. "Advertising injury" arising out of:

(1) Breach of contract. other than misappropriation of advertising ideas under an implied contract:

(2) The failure of goods. products or services to conform with advertised quality or performance:

(3) The wrong description of the price of goods, products or services; or

(4) An offense committed by an insured whose business is advertising. broadcasting. publishing or telecasting.

\section{COVERAGE C. MEDICAL PAYMENTS}

1. Insuring Agreement.

a. We will pay medical expenses as described below for "bodily injury" caused by an accident:

(1) On premises you own or rent: (2) On ways next to premises you own or
rent: or

(3) Because of your operations:

provided that:

(1) The accident takes place in the "coverage territory" and during the policy period:

(2) The expenses are incurred and reported to us within one year of the date of the 3ccident: and

(3) The injured person submits to examin ation. at our expense. by physicians ot our choice as often as we reasonably require.

b. We will makn these payments regardless of tault. These payments will not exceed the applicable himit of insurance We will pay reasonable expenses for:

(1) First aid at the time of an accident:

(2) Necessary medical, surgical, x-ray and dental services. including prosthetic devices: and

(3) Necessary ambulance, hospital, professional nursing and funeral services.

2. Exclusions.

We will not pay expenses for "bodily injury

a. To any insured.

b. To a person hired to do work for or on behalf of any insured or a tenint of any insured c. To a person injured on that part of premises you own or rent that the person normathy

d. To a person. whether or not an employee of any insured, if benefits for the "bodily injury" are payable or must be provided under a workers compensation or disability benefits law or a similar law.

- To a person injured while taking part in athletics.

f. Included within the "products-completed operations hazard."

g. Excluded under Coverage A.

h. Due to war, whether or not declared, or any act or condition incident to war. War includes civil war, insurrection, rebellion or revolution.

\section{SUPPLEMENTARY PAYMENTS - COVER.} AGES A AND B

We will pay. with respect to any claim or "suit" we defend:

1. All expenses we incur.

2. Up to $\$ 250$ for cost of bail bonds required because of accidents or traffic law violations arising out of the use of any vehicle to which the Bodily Injury Liability Coverage applies. We do not have to furnish these bonds.

3. The cost of bonds to release attachments, but only for bond amounts within the applicable limit of insurance. We do not have to furnish these bonds.

4. All reasonable expenses incurred by the insured at our request to assist us in the investigation or defense of the claim or "suit." including actuat loss of earnings up to $\$ 100$ a day because of time off from work.

5. All costs taxea against the insured in the "suit."

6. Pre-judgment nterest awarded against the in. sured on that part of the judgment we pay. If we make an offer :o pay the applicable limit of insur. ance. we will not pay any pre-judgment interest based on that period of time after the offer.

7. All interest on the full amount of any judgment that accrues after entry of the judgment and before we have paid, offered to pay. or deposited in court the part of the judgment that is within the applicable limit of insurance.

These payments will not reduce the limits of insur. ance.

\section{SECTION II - WHO IS AN INSURED}

1. If you are desigrated in the Declarations as

a. An individua. you and your spouse are in. sureds. but only with respect to the conduct of a business of which you are the sole owner

P.age 4 of 11 
b. A partnership or joint venture, you are an insured. Your members, your partners, and their spouses are also insureds, but only with respect to the conduct of your business.

c. An organization other than a partnership or joint venture, you are an insured. Your executive officers and directors are insureds. but only with respect to their duties as your officers or directors. Your stockholders are also insureds, but only with respect to their liability as stockholders.

2. Each of the following is also an insured:

a. Your employees, other than your executive officers, but only for acts within the scope of their employment by you. However, none of these employees is an insured for:

(1) "Bodily injury" or "personal injury" to you or to a co-employee while in the course of his or her employment; or

(2) "Bodily injury" or "personal injury" aris. ing out of his or her providing or failing to provide professional health care services: or

(3) "Property damage" to property owned or occupied by or rented or loaned to that employee, any of your other employees. or any of your partners or members (if you are a partnership or joint venture)

b. Any person (other than your employee) or any organization while acting as your real estate manager.

c. Any person or organization having proper temporary custody of your property if you die. but only

(1) With respect to liability arising out of the maintenance or use of that property: and

(2) Until your legal representative has been appointed.

d. Your legal representative if you die. but only with respect to duties as such. That represen tative will have all your rights and duties under this Coverage Part.

3. With respect to "mobile equipment" registered in your name under any motor vehicle registration law, any person is an insured while driving such equipment along a public highway with your permission. Any other person or organization responsible for the conduct of such person is also an insured. but only with respect to liability arising out of the operation of the equipment. and only if no other insurance of any kind is available to that person or organization for this liability. However. no person or organization is an insured with respect to

a. "Bodily injury" to a co-employee of the person driving the equipment: or

\section{COMMERCIAL GENERAL LIABIUTY}

COVERAGE FORM

b. "Property damage" to property owned by. rented to, in the charge of or occupied by you or the employer of any person who is an insured under this provision.

4. Any organization you newly acquire or form, other than a partnership or joint venture which you maintain ownership or maiter and over est. will be deemed to be a Named majority inter is no other similar insura Named insured it there is no other similar insurance available to that organization. However:

a. Coverage under this provision is afforded onty until the 9oth day after you acquire or form the organization or the end of the policy period. whichever is earlier:

b. Coverage A does not apply to "bodily injury" or "property damage" that occurred before you acquired or formed the organization; and

c. Coverage $\mathbf{B}$ does not apply to "personal injury" or "advertising injury" arising out of an offense committed before you acquired or formed the organization.

No person or organization is an insured with respect to the conduct of any current or past partnership or joint venture that is not shown as a Named insured in the Declarations.

\section{SECTION III - LIMITS OF INSURANCE}

1. The Limits of Insurance shown in the Declarations and the rules below fix the most we will pay regardless of the number of:

a. Insureds:

b. Claims made or "suits" brought: or

c. Persons or organizations making claims or bringing "suits."

2. The General Aggregate Limit is the most we will pay for the sum of:

a. Medical expenses under Coverage $C$ : and

b. Damages under Coverage A and Coverage B. except damages because of injury and dam. age included in the "products-completed operations hazard."

3. The Products-Completed Operations Aggregate Limit is the most we will pay under Coverage A for damages because of injury and damage included in the "products-completed operations hazard."

4. Subject to 2. above, the Personal and Advertising Injury Limit is the most we will pay under Cover. age $B$ for the sum of all damages because of all "personal injury" and all "advertising injury" sustained by any one person or organization.

5. Subject to 2 or 3 above, whichever applies, the Each Occurrence Lımit is the most we will pay for the sum of

a. Damages under Coverage $A$ : and

CG 00020286 


\section{COMMERCIAL GENERAL LIABILITY}

\section{COVERAGE FORM}

\section{b. Medical expenses under Coverage $C$} because of all "bodily injury" and "property damage" arising out of any one "occurrence."

6. Subject to 5. above, the Fire Damage Limit is the most we will pay under Coverage $A$ for damages because of "property damage " to premises rented to you arising out of any one fire.

7. Subject to 5. above. the Medical Expense Limit is the most we will pay under Coverage $C$ for all medical expenses because of "bodily injury" sustained by any one person.

The limits of this Coverage Part apply separately to each consecutive annual period and to any remaining period of less than 12 months, starting with the beginning of the policy period shown in the Declarations. unless the policy period is extended after issuance for an additional period of less than 12 months. case, the additional period will be deemed part of the last precedingeriod for purposes of determ of the last preceding period for purposes of determining the Limits of Insurance.

\section{SECTION IV - COMMERCIAL GENERAL}

\section{LIABILITY CONDITIONS}

1. Bankruptcy.

Bankruptcy or insolvency of the insured or of the insured's estate will not relieve us of our obliga. tions under this Coverage Part.

2. Duties In The Event Of Occurrence, Claim Or Suit.

a. You must see to it that we are notified as soon as practicable of an "occurrence" which may result in a claim. To the extent possible. notice should include:

(1) How, when and where the "occurrence" took place:

(2) The names and addresses of any injured persons and witnesses: and

(3) The nature and location of any injury or damage arising out of the ".occurrence."

Notice of an "occurrence" is not notice of a claim.

b. If a claim is received by any insured you must

(1) Immediately record the specifics of the claim and the date received: and

(2) Notify us as soon as practicable

You must see to it that we receive written notice of the claim as soon as practicable.

c. You and any other involved insured must:

(1) Immediately send us copies of any demands, notices, summonses or legal pers received in connection with the claim or a "suit:"

(2) Authorize us to obtain records and other information:

(3) Cogperate with us in the investigation sefflement or defense of the claim or suit." and
(4) Assist us, upon our request, in the en forcement of any right against any person or organization which may be liable to the insured because of injury or damage to which this insurance may also apply.

d. No insureds will, except at their own cost voluntarily make a payment, assume any obligation, or incur any expense, other than for first aid. without our consent.

3. Legal Action Against Us.

No person or organization has a right under this Coverage Part:

a. To join us as a party or otherwise bring us into a "suit" asking for damages from an insured: or

b. To sue us on this Coverage Part unless all of its terms have been fully complied with.

A person or organization may sue us to recover on an agreed settlement or on a final judgment against an insured obtained after an actual trial: but we will not be liable for damages that are not payable under the terms of this Coverage Part or that are in excess of the applicable limit of insur. ance. An agreed settlement means a settlement and release of liability signed by us, the insured and the claimant or the claimant's legal represen tative.

4. Other Insurance.

If other valid and collectible insurance is available to the insured for a loss we cover under Coverages A or $B$ of this Coverage Part, our obligations are limited as follows:

a. Primary Insurance

This insurance is primary except when b. below applies. If this insurance is primary, our obligations are not affected unless any of the other insurance is also primary. Then, we will share with all that other insurance by the method described in $\mathrm{c}$. below.

b. Excess Insurance

This insurance is excess over any of the other insurance. whether primary, excess, con. tingent or on any other basis:

(1) That is effective prior to the beginning of the policy period shown in the Declarations of this insurance and applies to "bodily injury" or "property damage" on other than a claims-made basis, if:

(a) No Retroactive Date is shown in the Declarations of this insurance; or

(b) The other insurance has a policy period which continues after the Retroactive Date shown in the Declarations of this insurance: 


\section{COMMERCIAL GENERAL WMBILITY}

COVERAGE FORM

(2) That is Fire, Extended Coverage, Builders' Risk. Installation Risk or similar coverage for "your work:"

(3) That is Fire insurance for premises rented to you: or

(4) If the loss arises out of the maintenance or use of aircraft. "autos" or watercraft to the extent not subject to Exclusion g. of Coverage A (Section I).

When this insurance is excess. we will have no duty under Coverages A or B to defend any claim or "suit" that any other insurer has a duty to defend. If no other insurer defends. we will under take to do so. but we will be entitled to the insured's rights against all those other insurers.

When this insurance is excess over other insurance. we will pay only our share of the sum of:

(1) The total amount that all such other insurance would pay for the loss in the absence of this insurance: and

(2) The total of all deductible and self-insured amounts under all that other insurance. We will share the remaining loss, if any. with any other insurance that is not described in this Excess insurance provision and was in bought specifically to apply in excess of the Limits of Insurance shown in the Declarations of this Coverage Part.

c. Method of Sharing

If all of the other insurance permits contribution by equal shares. we will follow this meth od also. Under this approach each insurer contributes equal amounts until it inas parer applicable limit of insurance or none of the loss remains. whichever comes first.

If any of the other insurance does not permit contribution by equal shares. we will con. tribute by limits. Under this method. each insurer's share is based on the ratio of its applicable limit of insurance to the total appli. cable limits of insurance of all insurers.

5. Premium Audit

a. We will compute all premiums for this Cover. age Part in accordance with our rules and rates.

b. Premium shown in this Coverage Part as advance premium is a deposit premium only At the close of each audit period we will com pute the earned premium for that period Audit premiums are due and payable on no tice to the first Named Insured. If the sum of the udvance and audit premiums paid for the 
COMMERCIAL GENERAL LIABILITY COVERAGE FORM

b. A summary by policy year, of payments made and amounts reserved, stated separately. under any applicable General Aggregate Limit and Products-Completed Operations Aggre gate Limit.

Amounts reserved are based on our judgment. They are subject to change and should not be regarded as uitimate settlement values.

If we cancel or elect not to renew this Coverage Part. we will provide such information no later than 30 days before the date of policy termina. tion. In other circumstances. we will provide this information only if we receive a written request from the first Named insured within 60 days after the end of the policy period. In this case, we will provide this information within $\mathbf{4 5}$ days of receipt of the request.

"We compile claim and "occurrence" information for our own business purposes and exercise reasonable care in doing so. In providing this information to the first Named Insured, we make no representations or warranties to insureds. insurers. or others to whom this information is furnished by or on behalf of any insured. Cancella. tion or non-renewal will be effective even if we inadvertently provide inaccurate information.

\section{SECTION V - EXTENDED REPORTING PE.}

\section{RIODS}

1. We will provide one or more Extended Reporting Periods, as described below, if:

a. This Coverage Part is cancelled or not renewed: or

b. We renew or replace this Coverage Part with insurance that:

(1) Has a Retroactive Date later than the date shown in the Declarations of this Coverage Part. or

(2) Does not upply to "bodily injury" or "property damage" on a claims-made basis

2. A Basic Extended Reporting Period is automatically provided without additional charge. This period starts with the end of the policy period and lasts for:

a. Five years for claims arising out of an "occurrence" reported to us, not later than 60 days after the end of the policy period. in accord. ance with paragraph 2.a. of SECTION IV COMMERCIAL GENERAL LIABILITY CONDI. TIONS. or

b. Sixty days for all other claims

The Basic Extended Reporting Period does not apply to cl.ams that are covered under any subse. quent insurance you purchase. or that would be covered but for exhaustion of the amount of insur. ance applicable to such claims

Page 8 of 11

Copyright, Insurance Services Office. Inc 1982.1986
3. A Supplemental Extended Reporting Period of unlimited duration is available, but only by an endorsement and for an extra charge. This supplemental period starts:

a. Five years after the end of the policy period for claims arising out of an "occurrence" reported to us, not later than 60 days after the end of the policy period, in accordance with paragraph 2.a. of SECTION IV - COMMERCIAL GENERAL LIABILITY CONDITIONS: or

b. Sixty days after the end of the policy period for all other claims.

You must give us a written request for the endorsement within 60 days after the end of the policy period. The Supplemental Extended Reporting Period will not go into effect unless you pay the additional premium promptly when due. We will determine the additional premium in accordance with our rules and rates. In doing so. we may take into account the following:

a. The exposures insured:

b. Previous types and amounts of insurance:

c. Limits of Insurance available under this Cov. erage Part for future payment of damages: and

d. Other related factors.

The additional premium will not exceed $200 \%$ of the annual premium for this Coverage Part.

This endorsement shall set forth the terms. not inconsistent with this Section, applicable to the Supplemental Extended Reporting Period. including a provision to the effect that the insurance afforded for claims first received during such period is excess over any other valid and collectible insurance available under policies in force after the Supplemental Extended Reporting Peri. od starts.

4. Extended Reporting Periods do not extend the policy period or change the scope of coverage provided. They apply only to claims for "bodily injury" or property damage" that occurs before the end of the policy period (but not before the Retroactive Oate. if any. shown in the Declara. tions).

Claims for such injury or damage which are fırst received and recorded during the Basic Extended Reporting Period (or during the Supplemental Extended Reporting Period, if it is in effect) will be deemed to have been made on the last day of the policy period

Once in effect. Extended Reporting Periods may not be cancelled 
5. Extended Reporting Periods do not reinstate or increase the Limits of Insurance applicable to any claim to which this Coverage Part applies, except to the extent described in paragraph 6 . of this Section.

6. If the Supplemental Extended Reporting Period is in effect. we will provide the separate aggregate limits of insurance described below, but only for claims first received and recorded during the Sup. plemental Extended Reporting Period.

The separate aggregate limits of insurance will be equal to the dollar amount shown in the Declara. tions in effect at the end of the policy period for such of the following limits of insurance for which a dollar amount has been entered:

\section{General Aggregate Limit}

Products-Completed Operations Aggregate Limit

Paragraphs 2. and 3. of SECTION III - LIMITS OF INSURANCE will be amended accordingly. The Each Occurrence Limit and the Fire Damage Limit shown in the Declarations will then continue to apply, as set forth in paragraphs 5 . and 6 . of that Section.

\section{SECTION VI - DEFINITIONS}

1. "Advertising injury" means injury arising out of one or more of the following offenses:

a. Oral or written publication of material that slanders or libels a person or organization or disparages a person's or organization's goods. products or services:

b. Oral or written publication of material that violates a person's right of privacy:

c. Misappropriation of advertising ideas or style of doing business: or

d. Intringement of copyright, title or slogan.

2. "Auto" means a hand motor vehicle, traler or semitraler designed for travel on public roads. including any attached machinery or equipment. But "auto" does no: include "mobile equipment."

3. "Bodily Injury" means bodily injury. sickness or Jisease sustained by a person. including death resulting from any of these at any time.

4. "Coverage territory" means

a. The United States of America (including its territories and possessions). Puer to Rico and Canada:

b. international waters or airspace. provided the infury or damage does not ociur in the course of travel or transportation to or from any slace not included in a above. or

c. Alt parts of the world it

\section{COMMERCIAL GENERAL LIABIUTY} COVERAGE FORM

(1) The injury or damage arises out of:

(a) Goods or products made or sold by you in the territory described in a. above; or

(b) The activities of a person whose home is in the territory described in a. above, but is away for a short time on your business: and

(2) The insured's responsibility to pay damages is determined in a "suit" on the merits, in the territory described in a. above or in a settlement we agree to.

5. "Impaired property" means tangible property. other than "your product" or "your work." that cannot be used or is less useful because:

a. It incorporates "your product" or "your work" that is known or thought to be defec. tive. deficient. inadequate or dangerous: or

b. You have failed to fulfill the terms of a contract or agreement:

if such property can be restored to use by:

a. The repair, replacement, adjustment or removal of "your product" or "your work:" or

b. Your fulfilling the terms of the contract or agreement.

6. "Insured contract" means:

a. A lease of premises:

b. A sidetrack agreement:

c. An easement or license agreement in connec. tion with vehicle or pedestrian private railroad crossings at grade:

d. Any other easement agreement. except in connection with construction or demolition operations on or within $\mathbf{5 0}$ feet of a railroad:

e. An indemnification of a municipality as requir. ed by ordinance, except in connection with work for a munucipality:

f. An elevator maintenance agreement: or

g. That part of any other contract or agreement pertaining to your business under which you assume the tort liability of another to pay damages because of "bodily injury" o: "prop. erty damage" to a third person or organiza. tion, if the contract or agreement is made prior to the "bodily injury" or "property dam. age." Tort liability means a liability that would be imposed by law in the absence of any con. tract or agreement.

An "insured contract" does not include that par:

of any contract or agreement

a. That indemuifies an atchitect, engineer sut veyo: tor injury or tamage arising out of 
COMMERCIAL GENERN LIABILITY COVERAGE FORM

(1) Preparing. approving or failing to prepare or approve maps, drawings, opinions, reports, surveys, change orders, designs or specifications; or

(2) Giving directions or instructions, or failing to give them, if that is the primary cause of the injury or damage:

b. Under which the insured, if an architect. engineer or surveyor, assumes liability for injury or damage arising out of the insured's rendering or failing to render professional services, including those listed in a. above and supervisory. inspection or engineering services; or

c. That indemnifies any person or organization for damage by fire to premises rented or loaned to you.

71 "Loading or unloading" means the handling of property:

a. After it is moved from the place where it is accepted for movement into or onto an aircraft. watercraft or "auto:"

b. While it is in or on an aircraft, watercraft or "auto:" or

c. While it is being moved from an aircraft. watercraft or "auto" to the place where it is finally delivered:

but "loading or unloading" does not include the movement of property by means of a mechanical device, other than a hand truck, that is not attached to the aircraft. watercraft or "auto."

8. "Mobile equipment" means any of the following types of land vehicles. including any attached machinery or equipment:

a. Bulldozers. farm machınery. forklifts and other venicles des:zned for use principally of public roads.

b. Vehicles maintained tor use solely on or nex to premises you own or rent:

c. Vehicles that travel on crawler treads:

d. Vehicles. whether self-propelled or not. main. tained primarily to provide mobility to perma. nently mounted

(1) Power cranes shovels, loaders. diggers or drills: or

(2) Road construction or resurfacing equipment such as graders. scrapers or rollers:

e. Vehicles not described in a.. b.. c. or d. above that are not self-propelled and are maintained primarily to provide mobility to permanently attached equipment of the following types.
(1) Air compressors, pumps and generators. including spraying. welding, building cleaning. geophysical exploration, lighting and well servicing equipment; or

(2) Cherry pickers and similar devices used to raise or lower workers:

f. Vehicles not described in a., b.. c. or d. above maintained primarily for purposes other than the transportation of persons or cargo.

However, self-propelled vehicies with the following types of permanently attached equipment are not "mobile equipment" but will be considered "autos:"

(1) Equipment designed primarily for:

(a) Snow removal;

(b) Road maintenance, but not construc. tion or resurfacing:

(c) Street cleaning:

(2) Cherry pickers and similar devices mounted on automobile or truck chassis and used to raise or lower workers; and

(3) Air compressors. pumps and generators including spraying. welding, building cleaning. geophysical exploration, light. ing and well servicing equipment.

9. "Occurrence" means an accident, including continuous or repeated exposure to substantially the same general harmful conditions.

10. "Personal injury" means injury. other than "bodily injury." arising out of one or more of the following offenses:

a. False arrest, detention or imprisonment:

b. Malicious prosecution

c. Wrongful entry into. or eviction of a person from, a room. dwelling or premises that the person occupies:

d. Oral or written publication of material that slanders or libels a person or organization or disparages a person's or organization's goods. products or services: or

e. Oral or written publication of material that violates a person's right of privacy.

11. a. "Products-completed operations hazard" includes all "bodily injury" and "property damage " occurring away from premises you own or rent and arising out of "your product" or "your work" except:

(1) Products that are still in your physical possession: or

(2) Work that has not yet been completed or abandoned

b. "Your work" will be deemed completed at the earliest of the following times 
(1) When all of the work called for in your contract has been completed.

(2) When all of the work to be done at the site has been completed if your contract calls for work at more than one site.

(3) When that part of the work done at a job site had been put to its intended use by any person or organization other than another contractor or subcontractor working on the same project.

Work that may need service, maintenance. correction, repair or replacement, but which is otherwise complete, will be treated as completed.

c. This hazard does not include "bodily injury" or "property damage" arising out of:

(1) The transportation of property, unless the injury or damage arises out of a condition in or on a vehicle created by the "loading or unloading" of it;

(2) The existence of tools, uninstalled equipment or abandoned or unused materials:

(3) Products or operations for which the classification in this Coverage Part or in our manual of rules includes products or completed operations.

12. "Property damage" means

a. Physical injury to tangible property, including all resulting loss of use of that property: or

b. Loss of use of tangible property that is not physically injured.

13. "Suit" means a civil proceeding in which damages because of "bodily injury." "property damage. " "personal injury " or " advertising injury " to which this nsurance applies are alleged. "Suit" includes an arbitration proceeding alleging such damages to which you must submit or submit with our co: sent

\section{COMMERCIAL GENERAL LIABIUTY} COVERAGE FORM

14. "Your product" means:

a. Any goods or products, other than real prop erty, manufactured, sold, handled, distrib uted or disposed of by:

(1) You:

(2) Others trading under your name; or

(3) A person or organization whose business or assets you have acquired: and

b. Containers (other than vehicles), materiais. parts or equipment furnished in connection with such goods or products.

"Your product" includes warranties or representations made at any time with respect to the fitness, quality, durability or performance of any of the items included in $a$. and b. above.

"Your product" does not include vending machines or other property rented to or located for the use of others but not sold.

15. "Your work" means:

a. Work or operations performed by you or on your behalf; and

b. Materials. parts or equipment furnished in connection with such work or operations.

"Your work" includes warranties or representations made at any time with respect to the fitness. quality. durability or performance of any of the items included in a. or b. above. 
